DESY 14-233

ISSN 0418-9833

November 2014

\title{
B-meson production in the Parton Reggeization Approach at Tevatron and the $\mathrm{LHC}$
}

\author{
A.V. Karpishkov* \\ Samara State University, Ac. Pavlov, 1, 443011 Samara, Russia \\ V.A. Saleevt \\ Samara State University, Ac. Pavlov, 1, 443011 Samara, Russia and \\ Samara State Aerospace University, \\ Moscow Highway, 34, 443086, Samara, Russia \\ M.A. Nefedov and A.V. Shipilova \\ Samara State University, Ac. Pavlov, 1, 443011 Samara, Russia \\ Samara State Aerospace University, \\ Moscow Highway, 34, 443086, Samara, Russia and \\ II. Institut für Theoretische Physik, Universität Hamburg, \\ Luruper Chaussee 149, 22761 Hamburg, Germany
}




\begin{abstract}
We study the inclusive hadroproduction of $B^{0}, B^{+}$, and $B_{s}^{0}$ mesons at leading order in the parton Reggeization approach using the universal fragmentation functions extracted from the combined $e^{+} e^{-}$annihilation data from CERN LEP1 and SLAC SLC colliders. We have described $B$-meson transverse momentum distributions measured in the central region of rapidity by the CDF Collaboration at Fermilab Tevatron and CMS Collaboration at LHC within uncertainties and without free parameters, applying Kimber-Martin-Ryskin unintegrated gluon distribution function in a proton. The forward $B$-meson production $(2.0<y<4.5)$ measured by the LHCb Collaboration also has been studied and expected disagreement between our theoretical predictions and data has been obtained.
\end{abstract}

PACS numbers: 12.38.-t,12.40.Nn,13.85.Ni,14.40.Gx

*Electronic address: karpishkov@rambler.ru

${ }^{\dagger}$ Electronic address: saleev@samsu.ru

${ }^{\ddagger}$ Electronic address: nefedovma@gmail.com

$\S$ Electronic address: alexshipilova@samsu.ru 


\section{INTRODUCTION}

The study of the heavy flavor production in high-energy hadronic interactions is well suited to solve a number of tasks in particle physics. At first, it provides a crucial test of the next-to-leading order (NLO) calculations in perturbative quantum chromodynamics (QCD) due to the smallness of strong coupling constant $\alpha_{S}(\mu)$, as the lowest limit of typical energy scale of the hard interaction $\mu$ is controlled by the bottom quark mass $m \gg \Lambda_{Q C D}$, where $\Lambda_{Q C D}$ is the asymptotic scale parameter of QCD. At second, one can check the performance of different approaches to resum higher-order QCD corrections.

The experimental study was started by the first $B$-meson measurements at the CERN $S \bar{p} p S$ collider operating at a center-of-mass energy of $\sqrt{S}=0.63 \mathrm{TeV}[1]$, followed in the Tevatron era by measurements of the CDF and D0 Collaborations at $\sqrt{S}=1.8 \mathrm{TeV}[2]$ and $\sqrt{S}=1.96 \mathrm{TeV}[3,4]$. The quite recent results were published by the CMS Collaboration for inclusive $B^{0}$ [6], $B^{+}$[5], and $B_{s^{-}}$[7]-meson production in proton-proton collisions at $\sqrt{S}=7 \mathrm{TeV}$ at the CERN Large Hadron Collider (LHC). All these measurements were implemented in the central region of rapidity, while the LHCb detector at LHC, dedicated to physics of $B$-decays, enables to measure observables of heavy flavor production in the forward rapidity region. In the Ref. [8] the $\mathrm{LHCb}$ team reported very recent results on $B^{0}, B^{+}$- and $B_{s}^{0}$-production in the form of transverse momentum and rapidity distributions. Furthermore, both at Tevatron and LHC, the single and pair production of bottom-flavored jets was measured and the $b$-quark cross sections were reconstructed. We successfully described the latter in the terms of Parton Reggeization Approach (PRA) in our previous works, see Ref. [9, 10]. But since the $b$-quark cross-section reconstruction is implemented through the observation of $B$-mesons decayed, to consider a $B$-meson production in the framework of PRA seems to be a good test of its convenience, completing our earlier investigations on open bottom production.

The proposal to apply the PRA in the field of heavy flavor production is caused by the fact that since the TeV-energy range is achieved, we enter a new dynamical regime, namely the high-energy Regge limit, characterized by the condition $\sqrt{S} \gg \mu \gg \Lambda_{Q C D}$, where the large coefficients of new type $\log ^{n}(\sqrt{S} / \mu)$ appear in all-order terms of perturbative QCD series, violating its convergence. Such a way, the new small parameter $x \sim \mu / \sqrt{S}$ need to be introduced and the terms proportional to $\log ^{n}(1 / x)$ should be resummed. 
The small- $x$ effects cause the distinction of the perturbative corrections relative for different processes and different regions of phase space. At first, the higher-order corrections for the production of heavy final states, such as Higgs bosons, top-quark pairs, dijets with large invariant masses, or Drell-Yan pairs, by initial-state partons with relatively large momentum fractions $x \sim 0.1$ are dominated by soft and collinear gluons and may increase the cross sections up to a factor 2. By contrast, relatively light final states, such as small-transversemomentum heavy quarkonia, single jets, prompt photons, or dijets with small invariant masses, are produced by the fusion of partons with small values of $x$, typically $x \sim 10^{-3}$ because of the large values of $\sqrt{S}$. Radiative corrections to such processes are dominated by the production of additional hard jets. The only way to treat such processes in the conventional collinear parton model $(\mathrm{CPM})$ is to calculate higher-order corrections in the strong coupling constant $\alpha_{S}=g_{S}^{2} / 4 \pi$, which could be a challenging task for some processes even at the next-to-leading order level. To overcome this difficulty and take into account a sizable part of the higher-order corrections in the small- $x$ regime, the $k_{T}$-factorization framework, was introduced [11-13].

The above mentioned $B$-meson production data at the LHC were been previously under study in the conventional collinear parton model of QCD at the next-to-leading order level of accuracy in the Refs. [14, 15], and for the discussion of Tevatron data see Refs. therein. The two working schemes were implemented: the general-mass variable-flavornumber (GM-VFN) scheme [16], and the so-called fixed order scheme improved with nextto-leading logarithms (FONLL scheme) [17]. In the former one, realized in the Ref. [14], the large fragmentation logarithms dominating at $p_{T}>m$ are resummed through the evolution of the nonperturbative fragmentation functions (FFs), satisfying the DokshitzerGribov-Lipatov-Altarelli-Parisi (DGLAP) [18] evolution equations. At the same time, the full dependence on the bottom-quark mass in the hard-scattering cross section is retained to describe consistently $p_{T} \sim m$ region. The $B$-meson FFs were extracted both at leading and next-to-leading order in the GM-VFN scheme from the combined fit of data on $B$-meson production in $e^{+} e^{-}$annihilation. Opposite, in the FONLL approach, the NLO $B$-meson production cross sections are calculated with a nonperturbative $b$-quark FF, that is not a subject to DGLAP [18] evolution. The FONLL scheme was implemented in the Refs. [15] and its main ingredients are the following: the NLO fixed order calculation (FO) with resummation of large transverse momentum logarithms at the next-to-leading level (NLL) for 
heavy quark production. For the consistency of the calculation, the NLL formalism should be used to extract the nonperturbative FFs from $e^{+} e^{-}$data, and in the Refs. [15] the scheme of calculation of heavy quark cross section and extraction of the nonperturbative FFs are directly connected and must be used only together. In general, the theoretical predictions obtained in Refs. [14, 15] describe data well within uncertainties.

The first study of open beauty hadroproduction in the alternative high-energy factorization scheme, namely the $k_{T}$-factorization framework [11 13], was firstly performed in the Ref. [19], where a good description of the $b \bar{b}$-pair production data from Tevatron, Run I, was acquired. The authors operated with off-shell initial gluons and the formalism of transverse-momentum dependent parton distributions. In the present work we develop this approach, introducing the $k_{T}$-factorization framework together with the fully gauge-invariant amplitudes with Reggeized gluons in the initial state. This combination we call the Parton Reggeization Approach everywhere below. We suppose PRA to be more theoretically reasonable than previous studies in $k_{T}$-factorization, as it is based on a gauge invariant effective theory for the QCD processes which occur in the quasi-multi-Regge kinematics. Therefore it preserves the gauge invariance of high-energy particle production amplitudes and allows a consistent continuation towards the NLO calculations.

Recently, PRA was successfully applied to analyze as the processes which involve heavy quark production: bottom-flavored jets [9, 10], charmonium and bottomonium production [20 24], as the number of others: inclusive production of single jet [25], pair of jets [26], prompt-photon [27, 28], photon plus jet [29], Drell-Yan lepton pairs [30], at Tevatron and the LHC. These studies have demonstrated the advantages of the high-energy factorization scheme used in PRA for the description of data, compared with the calculations in collinear parton model.

This paper is organized as follows. In Sec. II we present basic formalism of our calculations, the PRA and the fragmentation model. In Sec. III our results are presented in comparison with the experimental data and discussed. In Sec. IV we summarize our conclusions. 


\section{BASIC FORMALISM}

We study the production of $B$-mesons with high transverse momenta much larger than a $b$-quark mass. In this region we can apply the so-called massless scheme or zero-mass variable-flavor-number scheme (ZM-VFNS) [31, 32] treating a $b$-quark as a massless parton. For this case the $B$-cross section can be written in a factorized form as it stated by the factorization theorem of QCD [33]:

$$
\frac{d \sigma(p+p \rightarrow B+X)}{d p_{B T} d y}=\sum_{i} \int_{0}^{1} \frac{d z}{z} D_{i \rightarrow B}\left(z, \mu^{2}\right) \frac{d \sigma\left(p+p \rightarrow i\left(p_{i}\right)+X\right)}{d p_{i T} d y_{i}}
$$

where $D_{i \rightarrow B}\left(z, \mu^{2}\right)$ is the fragmentation function for producing the $B$-meson from the parton $i$, created at the hard scale $\mu$, the fragmentation parameter $z$ is defined through the relation $p_{i}=p_{B} / z$, with $p_{B}$ and $p_{i}$ to be $B$-meson and $i$-parton four-momenta, correspondingly, and their rapidities $y_{B}=y_{i}$. The high-transverse-momenta $b$-quark radiates a large amount of its energy in the form of hard, collinear gluons, causing the presence of the logarithms of the form $\alpha_{S} \log \left(\mu^{2} / m_{b}^{2}\right)$ in all orders of perturbative series. These large logarithms can be resummed through the Dokshitzer-Gribov-Lipatov-Altarelli- Parisi (DGLAP) evolution equations for nonperturbative fragmentation functions (FFs). The latter can be obtained only from experiment. In the Ref. [34], the nonperturbative FFs for the transitions $a \rightarrow$ $B$, where $a$ is any parton, including $b$ and $\bar{b}$ quarks, were extracted at NLO in the $\overline{M S}$ factorization scheme with $n_{f}=5$ flavors from the experimental data for the reaction $e^{+} e^{-} \rightarrow$ $B+X$ provided by the ALEPH [35] and OPAL [36] Collaborations at the CERN LEP1 collider and by the SLD Collaboration [37] at the SLAC SLC collider. These data were taken on the $Z$-boson peak, that strongly suppresses the finite- $m_{b}$ effects which are of relative order $m_{b}^{2} / m_{Z}^{2}$, giving the internal consistence of resulting FFs with the ZM-VFN scheme which we keep throughout our analysis. As input, in the fits of Refs. [34], the parameterizations at the initial scale $\mu_{0}=m_{b}$ for the FF's were taken in the simple power ansatz.

It was shown in Ref. [34], that the major part of $B$-mesons is produced through the gluon and bottom quark fragmentation, while the light quark fragmentation turns out to be negligible. Following this, in our study we will consider the $b$-quark and gluon fragmentation into different $B$-mesons only. To illustrate a difference of contributions to the $B$-meson production we show in the Fig. 3 the $b$-quark and gluon FF's into $B$-meson.

At high energies the bottom quarks are dominantly created via direct parton-parton col- 
lisions. When the center-of-mass energy is much larger than the bottom quark mass, the prior role is played by the gluon-gluon fusion. In hadron collisions the cross sections of processes with a hard scale $\mu$ can be represented as a convolution of scale-dependent parton (quark or gluon) distributions and squared hard parton scattering amplitude. These distributions correspond to the density of partons in the proton with longitudinal momentum fraction $x$ integrated over transverse momentum up to $k_{T}=\mu$. Their evolution from some scale $\mu_{0}$, which controls a non-perturbative regime, to the typical scale $\mu$ is described by DGLAP [18] evolution equations which allow to sum large logarithms of type $\log \left(\mu^{2} / \Lambda_{Q C D}^{2}\right)$ (collinear logarithms). The typical scale $\mu$ of the hard-scattering processes is usually of order of the transverse mass $m_{T}=\sqrt{m^{2}+\left|\mathbf{p}_{T}\right|^{2}}$ of the produced particle (or hadron jet) with (invariant) mass $m$ and transverse two-momentum $\mathbf{p}_{T}$. With increasing energy, when the ratio of $x \sim \mu / \sqrt{S}$ becomes small, the new large logarithms $\log (1 / x)$, soft logarithms, are to appear and can become even more important than the collinear ones. These logarithms present both in parton distributions and in partonic cross sections and can be resummed by the Balitsky-Fadin-Kuraev-Lipatov (BFKL) approach [38]. The approach gives the description of QCD scattering amplitudes in the region of large $S$ and fixed momentum transfer $t, S \gg|t|$ (Regge region), with various color states in the $t$-channel. Entering this region requires us to reduce approximations to keep the true kinematics of the process. It becomes possible introducing the unintegrated over transverse momenta parton distribution functions (UPDFs) $\Phi\left(x, t, \mu^{2}\right)$, which depend on parton transverse momentum $\mathbf{q}_{T}$ while its virtuality is $t=-\left|\mathbf{q}_{T}\right|^{2}$. The UPDFs are defined to be related with collinear ones through the equation:

$$
x G\left(x, \mu^{2}\right)=\int^{\mu^{2}} d t \Phi\left(x, t, \mu^{2}\right) .
$$

The UPDFs satisfy the BFKL evolution equation [38] which is suited to resum soft logarithms and appear in the BFKL approach as a particular result in the study of analytical properties of the forward scattering amplitude. The basis of the BFKL approach is the gluon Reggeization [39], as at small $x$ the gluons are the dominant partons.

The gluon Reggeization appears considering special types of kinematics of processes at high-energies. At large $\sqrt{S}$ the dominant contributions to cross sections of QCD processes gives multi-Regge kinematics (MRK). MRK is the kinematics where all particles have limited (not growing with $\sqrt{S}$ ) transverse momenta and are combined into jets with limited invariant 
mass of each jet and large (growing with $\sqrt{S}$ ) invariant masses of any pair of the jets. At leading logarithmic approximation of the BFKL approach (LLA), where the logarithms of type $\left(\alpha_{s} \log (1 / x)\right)^{n}$ are resummed, only gluons can be produced and each jet is actually a gluon. At next-to-leading logarithmic approximation (NLA) the terms of $\alpha_{s}\left(\alpha_{s} \log (1 / x)\right)^{n}$ are collected and a jet can contain a couple of partons (two gluons or quark-antiquark pair). Such kinematics is called quasi multi-Regge kinematics. Despite of a great number of contributing Feynman diagrams it turns out that at the Born level in the MRK amplitudes acquire a simple factorized form. Moreover, radiative corrections to these amplitudes do not destroy this form, and their energy dependence is given by Regge factors $s_{i}^{\omega\left(q_{i}\right)}$, where $s_{i}$ are invariant masses of couples of neighboring jets and $\omega\left(q_{i}\right)$ can be interpreted as a shift of gluon spin from unity, dependent from momentum transfer $q$. This phenomenon is called gluon Reggeization.

Due to the Reggeization of quarks and gluons, an important role is dedicated to the vertices of Reggeon-particle interactions. In particular, these vertices are necessary for the determination of the BFKL kernel. To define them we can notice the two ways: the "classical" BFKL method [40] is based on analyticity and unitarity of particle production amplitudes and the properties of the integrals corresponding to the Feynman diagrams with two particles in the $t$-channel has been developed. Alternatively, they can be straightforwardly derived from the non-Abelian gauge-invariant effective action for the interactions of the Reggeized partons with the usual QCD partons, which was firstly introduced in Ref. [41] for Reggeized gluons only, and then extended by inclusion of Reggeized quark fields in the Ref. [42]. The full set of the induced and effective vertices together with Feynman rules one can find in Refs. [42, 43].

Recently, an alternative method to obtain the gauge-invariant $2 \rightarrow n$ amplitudes with off-shell initial-state partons, which is mathematically equivalent to the PRA, was proposed in Ref. [44]. These $2 \rightarrow n$ amplitudes are extracted by using the spinor-helicity representation with complex momenta from the auxiliary $2 \rightarrow n+2$ scattering processes which are constructed to include the $2 \rightarrow n$ scattering processes under consideration. This method is more suitable for the implementation in automatic matrix-element generators, but for our study the use of Reggeized quarks and gluons is found to be simpler.

As we mentioned above, we will consider the $B$-meson production by only the $b$-quark and gluon fragmentation. The lowest order in $\alpha_{S}$ parton subprocesses of PRA in which gluon or 
b-quark are produced are the following: a gluon production via two Reggeized gluon fusion

$$
\mathcal{R}+\mathcal{R} \rightarrow g
$$

and the corresponding quark-antiquark pair production

$$
\mathcal{R}+\mathcal{R} \rightarrow b+\bar{b}
$$

where $\mathcal{R}$ are the Reggeized gluons.

According to the prescription of Ref. [43], the amplitudes of relevant processes (3) and (4) can be obtained from the Feynman diagrams depicted in Figs. 1 and 2, where the dashed lines represent the Reggeized gluons. Of course, the last three Feynman diagrams in Fig. 2 can be combined into the effective particle-Reggeon-Reggeon (PRR) vertex [43].

Let us define four-vectors $\left(n^{-}\right)^{\mu}=P_{1}^{\mu} / E_{1}$ and $\left(n^{+}\right)^{\mu}=P_{2}^{\mu} / E_{2}$, where $P_{1,2}^{\mu}$ are the fourmomenta of the colliding protons, and $E_{1,2}$ are their energies. We have $\left(n^{ \pm}\right)^{2}=0, n^{+} \cdot n^{-}=2$, and $S=\left(P_{1}+P_{2}\right)^{2}=4 E_{1} E_{2}$. For any four-momentum $k^{\mu}$, we define $k^{ \pm}=k \cdot n^{ \pm}$. The four-momenta of the Reggeized gluons can be represented as

$$
\begin{aligned}
& q_{1}^{\mu}=\frac{q_{1}^{+}}{2}\left(n^{-}\right)^{\mu}+q_{1 T}^{\mu}, \\
& q_{2}^{\mu}=\frac{q_{2}^{-}}{2}\left(n^{+}\right)^{\mu}+q_{2 T}^{\mu},
\end{aligned}
$$

where $q_{T}=\left(0, \mathbf{q}_{T}, 0\right)$ The amplitude of gluon production in fusion of two Reggeized gluons can be presented as scalar product of Fadin-Kuraev-Lipatov effective PRR vertex $C_{\mathcal{R} \mathcal{R}}^{g, \mu}\left(q_{1}, q_{2}\right)$ and polarization four-vector of final gluon $\varepsilon_{\mu}(p)$ :

$$
\mathcal{M}(\mathcal{R}+\mathcal{R} \rightarrow g)=C_{\mathcal{R} \mathcal{R}}^{g, \mu}\left(q_{1}, q_{2}\right) \varepsilon_{\mu}(p)
$$

where

$$
\begin{aligned}
C_{\mathcal{R} \mathcal{R}}^{g, \mu}\left(q_{1}, q_{2}\right) & =-\sqrt{4 \pi \alpha_{s}} f^{a b c} \frac{q_{1}^{+} q_{2}^{-}}{2 \sqrt{t_{1} t_{2}}}\left[\left(q_{1}-q_{2}\right)^{\mu}+\frac{\left(n^{+}\right)^{\mu}}{q_{1}^{+}}\left(q_{2}^{2}+q_{1}^{+} q_{2}^{-}\right)\right. \\
& \left.-\frac{\left(n^{-}\right)^{\mu}}{q_{2}^{-}}\left(q_{1}^{2}+q_{1}^{+} q_{2}^{-}\right)\right],
\end{aligned}
$$

$a$ and $b$ are the color indices of the Reggeized gluons with incoming four-momenta $q_{1}$ and $q_{2}$, and $f^{a b c}$ with $a=1, \ldots, N_{c}^{2}-1$ is the antisymmetric structure constants of color gauge group $S U_{C}(3)$. The squared amplitude of the partonic subprocess $\mathcal{R}+\mathcal{R} \rightarrow g$ is straightforwardly found from Eq. (7) to be

$$
\overline{|\mathcal{M}(\mathcal{R}+\mathcal{R} \rightarrow g)|^{2}}=\frac{3}{2} \pi \alpha_{s} \mathbf{p}_{T}^{2}
$$


The amplitude of the process (44) can be presented in a same way, as a sum of three terms $\mathcal{M}(\mathcal{R}+\mathcal{R} \rightarrow b+\bar{b})=\mathcal{M}_{1}+\mathcal{M}_{2}+\mathcal{M}_{3}$

$$
\begin{aligned}
\mathcal{M}_{1}= & -i \pi \alpha_{s} \frac{q_{1}^{+} q_{2}^{-}}{\sqrt{t_{1} t_{2}}} T^{a} T^{b} \bar{U}\left(p_{1}\right) \gamma^{\alpha} \frac{\hat{p}_{1}-\hat{q}_{1}}{\left(p_{1}-q_{1}\right)^{2}} \gamma^{\beta} V\left(p_{2}\right)\left(n^{+}\right)^{\alpha}\left(n^{-}\right)^{\beta}, \\
\mathcal{M}_{2}= & -i \pi \alpha_{s} \frac{q_{1}^{+} q_{2}^{-}}{\sqrt{t_{1} t_{2}}} T^{b} T^{a} \bar{U}\left(p_{1}\right) \gamma^{\beta} \frac{\hat{p}_{1}-\hat{q}_{2}}{\left(p_{1}-q_{2}\right)^{2}} \gamma^{\alpha} V\left(p_{2}\right)\left(n^{+}\right)^{\alpha}\left(n^{-}\right)^{\beta}, \\
\mathcal{M}_{3}= & 2 \pi \alpha_{s} \frac{q_{1}^{+} q_{2}^{-}}{\sqrt{t_{1} t_{2}}} T^{c} f^{a b c} \frac{\bar{U}\left(p_{1}\right) \gamma^{\mu} V\left(p_{2}\right)}{\left(p_{1}+p_{2}\right)^{2}}\left[\left(q_{1}-q_{2}\right)^{\mu}+\right. \\
& \left.\left(n^{-}\right)^{\mu}\left(q_{2}^{+}+\frac{q_{2}^{2}}{q_{1}^{-}}\right)-\left(n^{+}\right)^{\mu}\left(q_{1}^{-}+\frac{q_{1}^{2}}{q_{2}^{+}}\right)\right],
\end{aligned}
$$

where $T^{a}$ are the generators of the fundamental representation of the color gauge group $S U_{C}(3)$

The squared amplitudes can be presented as follows

$$
\begin{gathered}
\overline{|\mathcal{M}(\mathcal{R}+\mathcal{R} \rightarrow b+\bar{b})|^{2}}=256 \pi^{2} \alpha_{s}^{2}\left(\frac{1}{2 N_{c}} \mathcal{A}_{\mathrm{Ab}}+\frac{N_{c}}{2\left(N_{c}^{2}-1\right)} \mathcal{A}_{\mathrm{NAb}}\right) \\
\mathcal{A}_{\mathrm{Ab}}=\frac{t_{1} t_{2}}{\hat{t} \hat{u}}-\left(1+\frac{p_{2}^{+}}{\hat{u}}\left(q_{1}^{-}-p_{2}^{-}\right)+\frac{p_{2}^{-}}{\hat{t}}\left(q_{2}^{+}-p_{2}^{+}\right)\right)^{2} \\
\mathcal{A}_{\mathrm{NAb}}=\frac{2}{S^{2}}\left(\frac{p_{2}^{+}\left(q_{1}^{-}-p_{2}^{-}\right) S}{\hat{u}}+\frac{S}{2}+\frac{\Delta}{\hat{s}}\right)\left(\frac{p_{2}^{-}\left(q_{2}^{+}-p_{2}^{+}\right) S}{\hat{t}}+\frac{S}{2}-\frac{\Delta}{\hat{s}}\right) \\
-\frac{t_{1} t_{2}}{q_{1}^{-} q_{2}^{+} \hat{s}}\left(\left(\frac{1}{\hat{t}}-\frac{1}{\hat{u}}\right)\left(q_{1}^{-} p_{2}^{+}-q_{2}^{+} p_{2}^{-}\right)+\frac{q_{1}^{-} q_{2}^{+} \hat{s}}{\hat{t} \hat{u}}-2\right) \\
\Delta=\frac{S}{2}\left(\hat{u}-\hat{t}+2 q_{1}^{-} p_{2}^{+}-2 q_{2}^{+} p_{2}^{-}+t_{1} \frac{q_{2}^{+}-2 p_{2}^{+}}{q_{2}^{+}}-t_{2} \frac{q_{1}^{-}-2 p_{2}^{-}}{q_{1}^{-}}\right)
\end{gathered}
$$

Here the bar indicates averaging (summation) over initial-state (final-state) spins and colors, $t_{1}=-q_{1}^{2}=\left|\mathbf{q}_{1 T}\right|^{2}, t_{2}=-q_{2}^{2}=\left|\mathbf{q}_{2 T}\right|^{2}$, and

$$
\begin{aligned}
& \hat{s}=\left(q_{1}+q_{2}\right)^{2}=\left(p_{1}+p_{2}\right)^{2}, \\
& \hat{t}=\left(q_{1}-p_{1}\right)^{2}=\left(q_{2}-p_{2}\right)^{2}, \\
& \hat{u}=\left(q_{2}-p_{1}\right)^{2}=\left(q_{1}-p_{2}\right)^{2} .
\end{aligned}
$$

The squared amplitude (11) analytically coincide with the previously obtained in Ref. [11]. We checked that in the collinear limit, i.e. $q_{(1,2) T} \rightarrow 0$, the squared amplitude (11) after averaging over the azimuthal angles transforms to the squared amplitude of the corresponding 
parton subprocess in collinear model, namely $g+g \rightarrow b+\bar{b}$. We perform our analysis in the region of $\sqrt{S}, p_{T} \gg m_{b}$, that allows us to use zero-mass variable-flavor-number-scheme (ZM VFNS), where the masses of the charm quarks in the hard-scattering amplitude are neglected.

In the $k_{T}$-factorization, differential cross section for the $2 \rightarrow 1$ subprocess (3) has the form:

$$
\begin{aligned}
& \frac{d \sigma}{d y d p_{T}}(p+p \rightarrow g+X)=\frac{1}{p_{T}^{3}} \int d \phi_{1} \int d t_{1} \Phi\left(x_{1}, t_{1}, \mu^{2}\right) \Phi\left(x_{2}, t_{2}, \mu^{2}\right) \times \\
& \overline{|\mathcal{M}(\mathcal{R}+\mathcal{R} \rightarrow g)|^{2}}
\end{aligned}
$$

where $\phi_{1}$ is the azimuthal angle between $\mathbf{p}_{T}$ and $\mathbf{q}_{1 T}$.

Analogous formula for the $2 \rightarrow 2$ subprocess (44) can be written as

$$
\begin{array}{r}
\frac{d \sigma}{d y_{1} d y_{2} d p_{1 T} d p_{2 T}}\left(p+p \rightarrow b\left(p_{1}\right)+\bar{b}\left(p_{2}\right)+X\right)=\frac{p_{1 T} p_{2 T}}{16 \pi^{3}} \int d \Delta \phi \times \\
\int d \phi_{1} \int d t_{1} \Phi\left(x_{1}, t_{1}, \mu^{2}\right) \Phi\left(x_{2}, t_{2}, \mu^{2}\right) \frac{\overline{|\mathcal{M}(\mathcal{R}+\mathcal{R} \rightarrow c+\bar{c})|^{2}}}{\left(x_{1} x_{2} S\right)^{2}}
\end{array}
$$

where $x_{1}=q_{1}^{+} / P_{1}^{+}, x_{2}=q_{2}^{-} / P_{2}^{-}, \Delta \phi$ is the azimuthal angle between $\mathbf{p}_{1 T}$ and $\mathbf{p}_{2 T}$, the rapidity of the final-state parton with four-momentum $p$ is $y=\frac{1}{2} \ln \left(\frac{p^{+}}{p^{-}}\right)$. Again, we have checked a fact that in the limit of $t_{1,2} \rightarrow 0$, we recover the conventional factorization formula of the collinear parton model from (16) and (17).

The important ingredient of the our scheme is unintegrated gluon distribution function, which we take as one proposed by Kimber, Martin and Ryskin (KMR) [45]. These distributions are obtained introducing a single-scale auxiliary function which satisfies the unified BFKL/DGLAP evolution equation, where the leading BFKL logarithms $\alpha_{S} \log (1 / x)$ are fully resummed and even a major (kinematical) part of the subleading BFKL effects are taken into account. This procedure to obtain UPDFs requires less computational efforts than the precise solution of two-scale evolution equations such as, for instance, CiafaloniCatani-Fiorani-Marchesini equation [46], but we found it to be suitable and adequate to physics of processes under study.

The usage of the $k_{T}$-factorization formula and UPDFs with one longitudinal (light-cone) kinematic variable $(x)$ requires the Reggeization of the $t$-channel partons. Accordingly to Refs. [41, 42], Reggeized partons carry only one large light-cone component of the fourmomentum and, therefore, it's virtuality is dominated by the transverse momentum. Such 
kinematics of the $t$-channel partons corresponds to the MRK of the initial state radiation and particles, produced in the hard process. In our previous analysis [20-24] devoted to the similar processes of heavy meson production we proved that these UPDFs give the best description of the heavy quarkonium $p_{T}$-spectra measured at Tevatron [47] and the LHC [48].

As the contribution of gluon fragmentation at $\mu>\mu_{0}$ is initiated by the perturbative transition of gluons to $b \bar{b}$-pairs encountered by DGLAP evolution equations, the part of $b$-quarks produced in the subprocess (44) with their subsequent transition to $B$-mesons are already taken into account considering $B$-meson production via gluon fragmentation. The simplest way to avoid double counting is to effectively subtract this contribution by the imposing of the lower cut on $\hat{s}$ at the threshold of the production of the $b \bar{b}$ pair in (17), i.e $\hat{s}>4 m_{b}^{2}$. The precise study of double-counting terms and other finite-mass effects needs a separate consideration and can be a subject of our future works.

\section{RESULTS}

We consequently come to the comparison of our predictions for the cross section distributions with experimental data. To illustrate the rise of the signals of high-energy-asymptotic effects due to increasing of the collision energy, we start our analysis from the data collected for the $B^{+}$-mesons at the collision energy of $\sqrt{S}=1.96 \mathrm{TeV}$ by the CDF Collaboration at Fermilab Tevatron, Run II [3]. The $B^{+}$-mesons were produced in the central region of

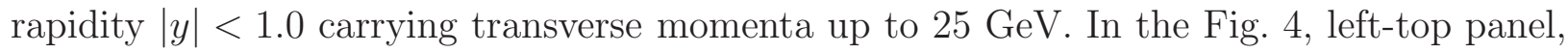
we introduce these data coming as differential cross sections $d \sigma / d p_{T}$, where the particle and antiparticle contributions are averaged, in comparison with our predictions in the LO of the PRA. The dashed lines represent contributions of the process (3) while dash-dotted lines correspond to ones of the process (44). The sum of both contributions is shown as a solid line. A theoretical uncertainty is estimated by varying factorization and renormalization scales between $1 / 2 m_{T}$ and $2 m_{T}$ around their central value of $m_{T}$, the transverse mass of a fragmenting parton. The resulting uncertainty is depicted in the figures by shaded bands. We follow our comparison increasing the collision energy but staying within the central rapidity region, turning to the description of the recent data from the LHC at $\sqrt{S}=7 \mathrm{TeV}$

collected by the CMS Collaboration for $B^{0}$ mesons at $|y|<2.2[6], B^{+}$and $B_{s}^{0}$ mesons at 
$|y|<2.4[5$, 7]. In the Fig. 4, right-top, left-bottom and right-bottom panels, we show the $p_{T^{-}}$distributions for $B^{0}, B^{+}$, and $B_{s}^{+}$mesons, correspondingly. At both collision energies considered we find a good agreement between our predictions and experimental data for the large values of $B$-meson transverse momenta, within experimental and theoretical uncertainties, while in the lower $p_{T}$ range our predictions are found to overshoot the data, except the $B_{s}^{+}$meson case, where a nice coincidence for all values of $p_{T}$ is obtained. But since we neglect the $b$-quark mass, the predictions in the region $p_{T} \sim m_{b}$ are obviously expected to overestimate the data and therefore should not confuse the reader. Comparing with the previous investigations at the NLO level of CPM, our results obtained at the LO of PRA nearly coincide with the recent ones derived in the framework of GM-VFN scheme [14, 34]. Considering the relative contributions of the subprocesses, in general we find the MRK and QMRK subprocesses to give equal contributions to $B$-meson production.

Finally, in the Fig. 5 we present our predictions for the planned LHC energy of $\sqrt{S}=$ $14 \mathrm{TeV}$ and keeping the other kinematic conditions as in the Refs. [5] 7].

Not only the central but also the forward rapidity region in $p p$ collisions at the LHC became available by the specially designed $\mathrm{LHCb}$ detector where the measurements of differential cross sections of $B^{0}, B^{+}$, and $B_{s}^{0}$ mesons including their charge-conjugate states were performed at $\sqrt{S}=7 \mathrm{TeV}$ with $2.0<y<4.5$ [8]. The observed data divided into 5 rapidity regions previously found a successful description in the FONLL scheme [15]. We present these data coming as double-differential distribution for the each of rapidity regions and the transverse momenta distribution integrated over all considered rapidities, together with our results obtained in the LO of PRA in the Figs. 6H [1] Nevertheless the PRA formalism discussed here is justified for the particle production in the central interval of rapidities, we obtain a good description of the data at $p_{T} \geq 10 \mathrm{GeV}$ even in the forward rapidity region. As for the region of the transverse momenta comparable to a $b$-quark mass, at the lower forward rapidities our predictions overestimate the experimental data. Moving towards the higher rapidities this excess transforms to the underestimation due to the strong decreasing of the contribution of subprocess (3) at small $p_{T}$ with increasing rapidity. This behaviour of gluon contribution differs the overall picture at forward rapidities from the central one, where the contributions of both subprocesses are approximately equal.

This effect can be explained if we we recall that with grow of rapidity of the particle produced in the hard scattering process the fraction of longitudinal momenta of initial 
proton transferred to this process increases simultaneously. That means that we enter the region of large $x>0.1$ where the conditions of Reggeization are not satisfied and the CPM should be applied instead of PRA. The large positive rapidity of a produced particle is provided by a large fraction $x$ from the hadron moving along the positive direction, and balanced by a very small fraction of negative longitudinal momenta carrying by the second hadron in the collision, up to $10^{-5}$. That leads to the situation in which we finely take into account small- $x$ effects although loosing in large- $x$. The region of large $x$ and small $p_{T}$ is a field of study for CPM, where the $2 \rightarrow 1$ processes with non-zero transverse momentum of final particle do not exist and one should start from $2 \rightarrow 2$ processes. Such a way, the contribution of the subprocess (3) falls down, and the subprocess (44) starts to give a dominant contribution, while the underestimating of the data is connected with the NLO corrections to the latter which are beyond this study. Moreover, as one can find from Fig. 3. the $b$-quark fragmentation function strongly exceeds the gluon one, especially at low $\mu^{2}$. This fact is confirmed by our recent work [10], where the bottom quark multiplicity in a gluon jet for different $\mu^{2}$ was extracted.

\section{CONCLUSIONS}

In the present work we performed the study of $B^{0}, B^{+}$, and $B_{s}^{0}$-meson fragmentation production in proton-(anti)proton collisions with central rapidities at Tevatron Collider and LHC, and in the forward rapidity region for the LHC, in the framework of Parton Reggeization Approach. Here we take into account all the hard-scattering parton subprocesses appearing at the LO with Reggeized gluons in the initial state. Among them there is a $2 \rightarrow 1$ subprocess of gluon production via Reggeized-gluon fusion, which was considered at the first time during the studies of $B$-meson production. To describe the hard scattering stage we use the fully gauge invariant amplitudes introduced in the works of L. N. Lipatov and co-authors. The distributions of initial partons are taken in the form of unintegrated parton distribution functions proposed by Kimber, Martin and Ryskin, and the way of their definition is ideologically related to the above-mentioned amplitudes. To describe the nonperturbative transition of gluons and $b$-quarks created at the hard stage into the $B$-mesons we use the universal fragmentation functions obtained from the fit of $e^{+} e^{-}$annihilation data from CERN LEP1 and SLAC SLC colliders. We obtained a good agreement of our results 
for $B$-meson central-rapidity production comparing with experimental data from Tevatron and the LHC, especially at large transverse momenta. The achieved degree of agreement for the central rapidity region is the same as the one obtained by NLO calculations in the conventional collinear parton model. The predictions for the $B$-meson production at central rapidities for the expected LHC energy of $\sqrt{S}=14 \mathrm{TeV}$ are also presented. At the forward rapidities our results for the transverse-momentum $B$-meson distributions are found to diverge with experimental data provided by the LHCb Collaboration at LHC, that effect is . We describe $B$-meson production without any free parameters or auxiliary approximations.

\section{ACKNOWLEDGEMENTS}

The work of A. V. Shipilova and A. V. Karpishkov was partly supported by the Grant of President of Russian Federation No. MK-4150.2014.2. The work of M.A. Nefedov and V.A. Saleev was supported in part by the Russian Foundation for Basic Research through the Grant No. 14-02-00021. A. V. Shipilova is grateful to Prof. G. Kramer for the useful discussions, to Prof. B. A. Kniehl for the kind hospitality, and to the German Academic Exchange Service (DAAD) together with the Russian Federal Ministry of Science and Education for the financial support by Grant No. A/13/75500.

[1] UA1 Collaboration, C. Albajar et al., Phys. Lett. B 213, 405 (1988).

[2] CDF Collaboration, F. Abe et al., Phys. Rev. Lett. 75, 1451 (1995); CDF Collaboration, D. E. Acosta et al., Phys. Rev. D 66, 052005 (2002); D0 Collaboration, S. Abachi et al., Phys. Rev. Lett. 74, 3548 (1995); D0 Collaboration, B. Abbott et al., Phys. Rev. Lett. 84, 5478 (2000).

[3] CDF Collaboration, A. Abulencia et al., Phys. Rev. D 75, 012010 (2007).

[4] CDF Collaboration, D. Acosta et al., Phys. Rev. D 71, 032001 (2005); CDF Collaboration, T. Aaltonen et al., Phys. Rev. D 79, 092003 (2009).

[5] CMS Collaboration, V. Khachatryan et al., Phys. Rev. Lett. 106, 112001 (2011).

[6] CMS Collaboration, S. Chatrchyan et al., Phys. Rev. Lett. 106, 252001 (2011).

[7] CMS Collaboration, S. Chatrchyan et al., Phys. Rev. D 84, 052008 (2011).

[8] LHCb Collaboration, R. AAij et al., JHEP 1308, 117 (2013). 
[9] B. A. Kniehl, A. V. Shipilova, and V. A. Saleev, Phys. Rev. D 81, 094010 (2010).

[10] V. A. Saleev, A. V. Shipilova Phys. Rev. D 86, 034032 (2012).

[11] J. C. Collins and R. K. Ellis, Nucl. Phys. B 360, 3 (1991).

[12] L. V. Gribov, E. M. Levin, and M. G. Ryskin, Phys. Rep. 100, 1 (1983).

[13] S. Catani, K. M. Ciafaloni, and F. Hautmann, Nucl. Phys. B 366, 135 (1991).

[14] B. A. Kniehl, G. Kramer, I. Schienbein, and H. Spiesberger, Phys. Rev. D 84, 094026 (2011).

[15] M. Cacciari, S. Frixione, N. Houdeau, M. L. Mangano, P. Nason, and G. Ridolfi JHEP 1210, 137 (2012).

[16] G. Kramer, and H. Spiesberger, Eur. Phys. J. C 22, 289 (2001); 28, 495 (2003); 38, 309 (2004); B. A. Kniehl, G. Kramer, I. Schienbein, and H. Spiesberger, Phys. Rev. D 71, 014018 (2005); B. A. Kniehl, G. Kramer, I. Schienbein, and H. Spiesberger, Eur. Phys. J. C 41, 199 (2005).

[17] M. Cacciari, M. Greco, P. Nason. JHEP 05 (1998) 007.

[18] V. N. Gribov and L. N. Lipatov, Sov. J. Nucl. Phys. 15, 438 (1972) [Yad. Fiz. 15, 781 (1972)]; Yu. L. Dokshitzer, Sov. Phys. JETP 46, 641 (1977) [Zh. Eksp. Teor. Fiz. 73, 1216 (1977)]; G. Altarelli and G. Parisi, Nucl. Phys. B126, 298 (1977).

[19] Ph. Hagler, R. Kirschner, A. Schafer, L. Szymanowski, and O. V. Teryaev, Phys. Rev. D 62, $071502(2000)$.

[20] B. A. Kniehl, V. A. Saleev, and D. V. Vasin, Phys. Rev. D 73, 074022 (2006).

[21] B. A. Kniehl, V. A. Saleev, and D. V. Vasin, Phys. Rev. D 74, 014024 (2006).

[22] V. A. Saleev and D. V. Vasin, Phys. Rev. D 68, 114013 (2003); Phys. Atom. Nucl. 68, 94 (2005) [Yad. Fiz. 68, 95 (2005)].

[23] V. A. Saleev, M. A. Nefedov, A. V. Shipilova, Phys. Rev. D 85, 074013 (2012).

[24] M. A. Nefedov, V. A. Saleev, A. V. Shipilova, Phys. Rev. D 88, 014003 (2013).

[25] B. A. Kniehl, V. A. Saleev, A. V. Shipilova, E. V. Yatsenko, Phys. Rev. D 84, 074017 (2011).

[26] M. A. Nefedov, V. A. Saleev, A. V. Shipilova Phys. Rev. D D87 , 094030 (2013).

[27] V. A. Saleev, Phys. Rev. D 78, 034033 (2008).

[28] V. A. Saleev, Phys. Rev. D 78, 114031 (2008).

[29] B. A. Kniehl, M. A. Nefedov, V. A. Saleev, Phys. Rev. D 89, 114016 (2014).

[30] M. A. Nefedov, N. N. Nikolaev, V. A. Saleev Phys. Rev. D 87, 014022 (2013).

[31] J. Binnewies, B. A. Kniehl, and G. Kramer, Phys. Rev. D 58, 034016 (1998). 
[32] M. Cacciari and M. Greco, Nucl. Phys. B 421, 530 (1994).

[33] B. Mele, P. Nason, Nucl. Phys. B 361, 626 (1991).

[34] B. A. Kniehl, G. Kramer, I. Schienbein, and H. Spiesberger, Phys. Rev. D 77, 014011 (2008).

[35] ALEPH Collaboration, A. Heister et al., Phys. Lett. B 512, 30 (2001).

[36] OPAL Collaboration, G. Abbiendi et al., Eur. Phys. J. C 29, 463 (2003).

[37] SLD Collaboration, K. Abe et al., Phys. Rev. D 65, 092006 (2002); 66, 079905(E) (2002).

[38] E. A. Kuraev, L. N. Lipatov, and V. S. Fadin, Sov. Phys. JETP 44, 443 (1976) [Zh. Eksp. Teor. Fiz. 71, 840 (1976)]; I. I. Balitsky and L. N. Lipatov, Sov. J. Nucl. Phys. 28, 822 (1978) [Yad. Fiz. 28, 1597 (1978)].

[39] E. A. Kuraev, L. N. Lipatov, V. S. Fadin, Phys. Lett. B 60, 50 (1975).

[40] V. S. Fadin, R. Fiore, Phys. Rev. D 64 114012, (2001).

[41] L. N. Lipatov, Nucl. Phys. B 452, 369 (1995).

[42] L. N. Lipatov and M. I. Vyazovsky, Nucl. Phys. B 597, 399 (2001).

[43] E. N. Antonov, L. N. Lipatov, E. A. Kuraev, and I. O. Cherednikov, Nucl. Phys. B 721, 111 (2005).

[44] A. van Hameren, K. Kutak, and T. Salwa, Phys. Lett. B 727, 226 (2013).

[45] M. A. Kimber, A. D. Martin, and M. G. Ryskin, Phys. Rev. D 63, 114027 (2001).

[46] M. Ciafaloni, Nucl. Phys. B 296, 49 (1988); S. Catani, F. Fiorani, G. Marchesini, Phys. Lett. B 234 339, (1990); Nucl. Phys. B 336 18, (1990).

[47] CDF Collaboration, F. Abe et al., Phys. Rev. Lett. 79, 572 (1997); 79, 578 (1997); CDF Collaboration, T. Affolder et al., Phys. Rev. Lett. 85, 2886 (2000); CDF Collaboration, D. Acosta et al., Phys. Rev. D 71, 032001 (2005); CDF Collaboration, F. Abe et al., Phys. Rev. Lett. 75, 4358 (1995); CDF Collaboration, D. Acosta et al., ibid. 88, 161802 (2002); CDF Collaboration, V. M. Abazov et al., Phys. Rev. Lett. 94, 232001 (2005).

[48] ATLAS Collaboration, G. Aad et al., Phys. Rev. D 87, 052004 (2013); CMS Collaboration, S. Chatrchyan et al., Phys. Rev. D 83, 112004 (2011); LHCb Collaboration, R. Aaij et al., Eur. Phys. J. C 72, 2025 (2012).

[49] V. S. Fadin and L. N. Lipatov, Nucl. Phys. B 477, 767 (1996). 

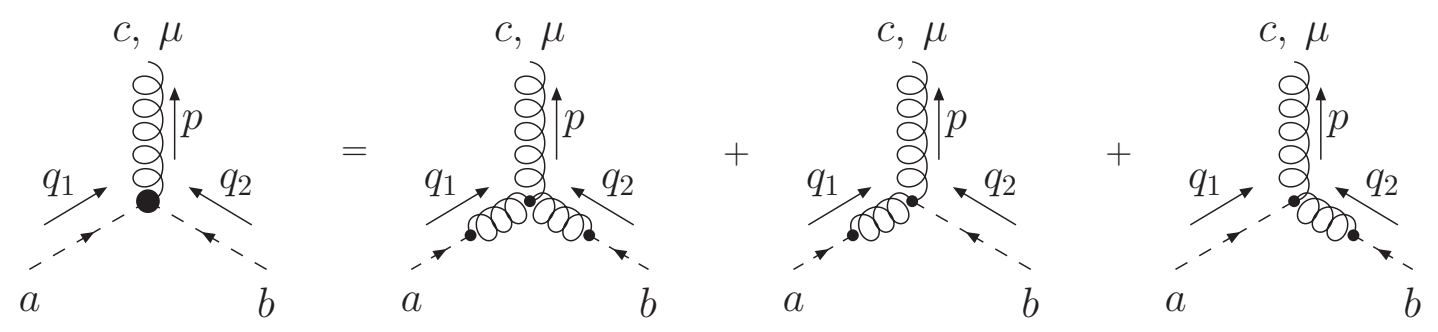

FIG. 1: Feynman diagrams for the subprocess (3).
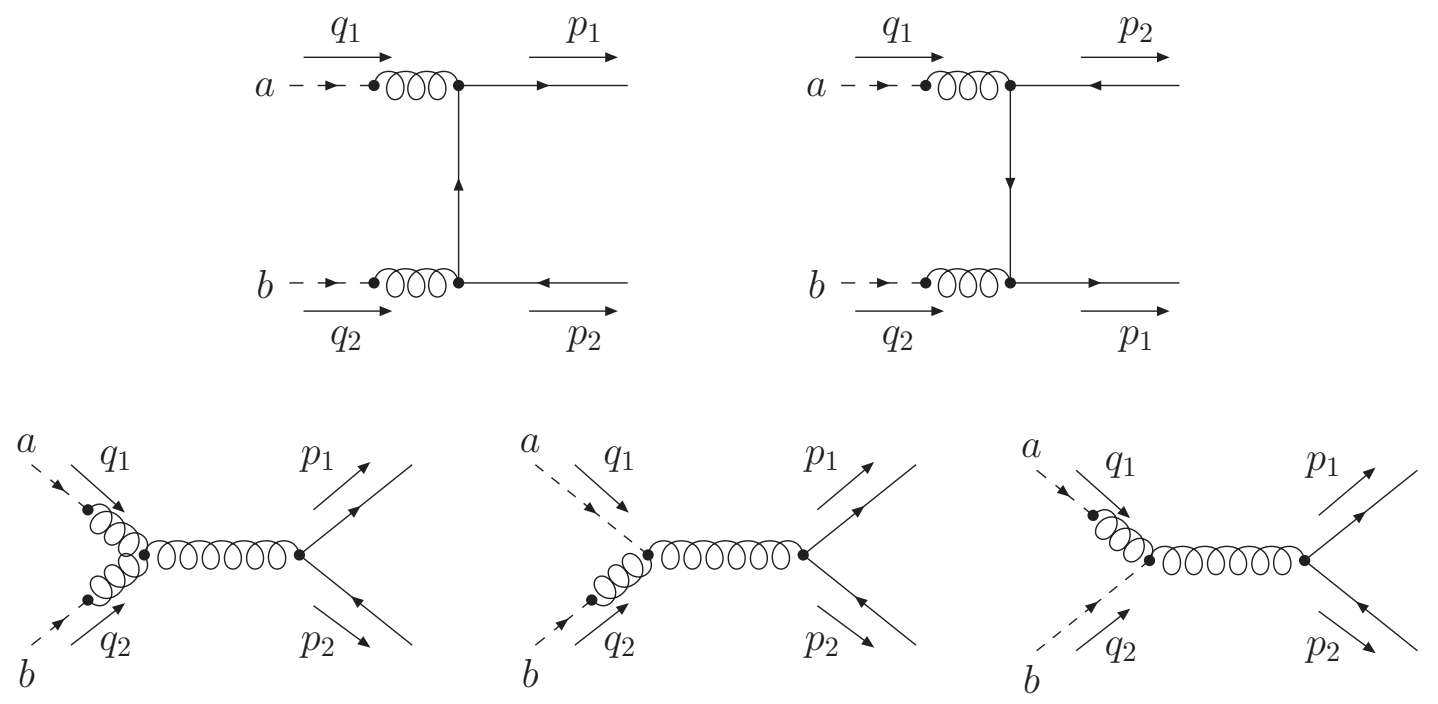

FIG. 2: Feynman diagrams for the subprocess (4). 


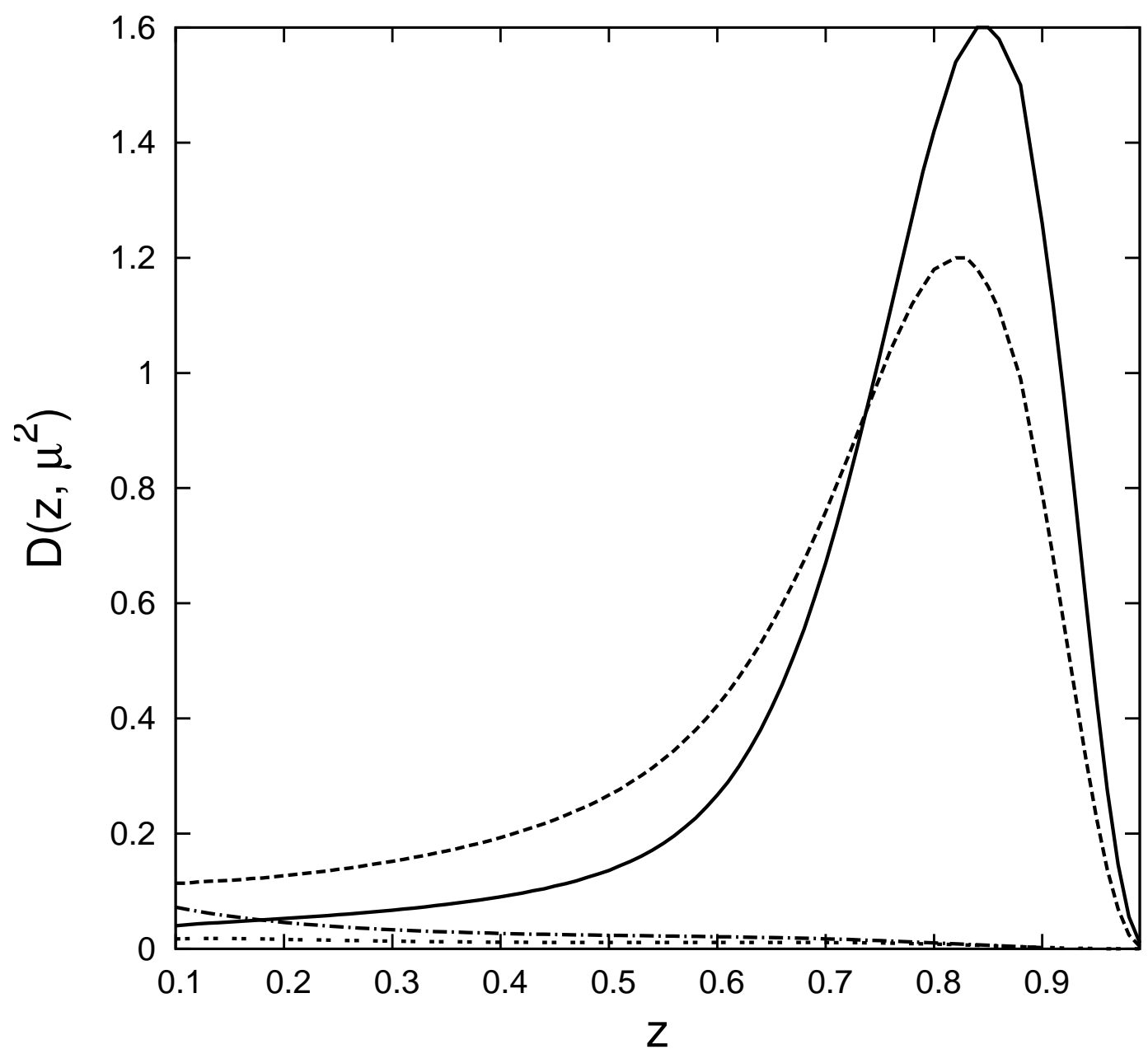

FIG. 3: The fragmentation function $D\left(z, \mu^{2}\right)$ of $b$-quarks and gluons into $B$ mesons from Ref. [34] at the $\mu^{2}=100 \mathrm{GeV}^{2}$ (solid curve for $b$-quark, pair-dotted for gluon) and $\mu^{2}=1000 \mathrm{GeV}^{2}$ (dashed line for $b$-quark, dash-dotted for gluon). 

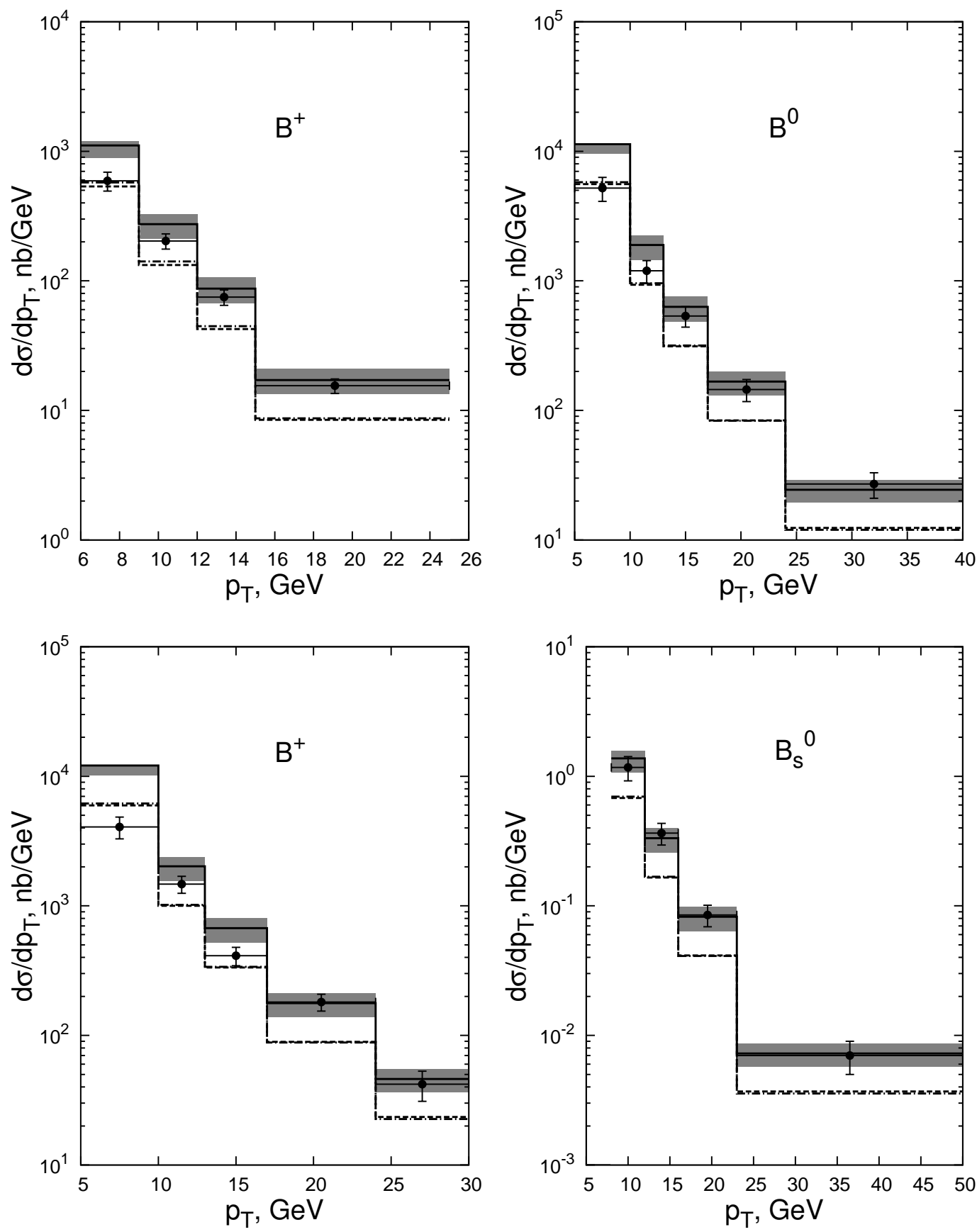

FIG. 4: Transverse momentum distributions of $B^{+}$-meson production at Tevatron, $\sqrt{S}=1.96 \mathrm{TeV}$ (left-top); $B^{0}$ (right-top), $B^{+}$(left-bottom), and $B_{s}^{0}$ (right-bottom) mesons at LHC, $\sqrt{S}=7 \mathrm{TeV}$. Dashed line represents the contribution of gluon fragmentation, dash-dotted line - the $b$-quarkfragmentation contribution, solid line is their sum. The CDF data at Tevatron are from the Ref. [3], the CMS data at LHC are from the Refs. [5-7], correspondingly. 

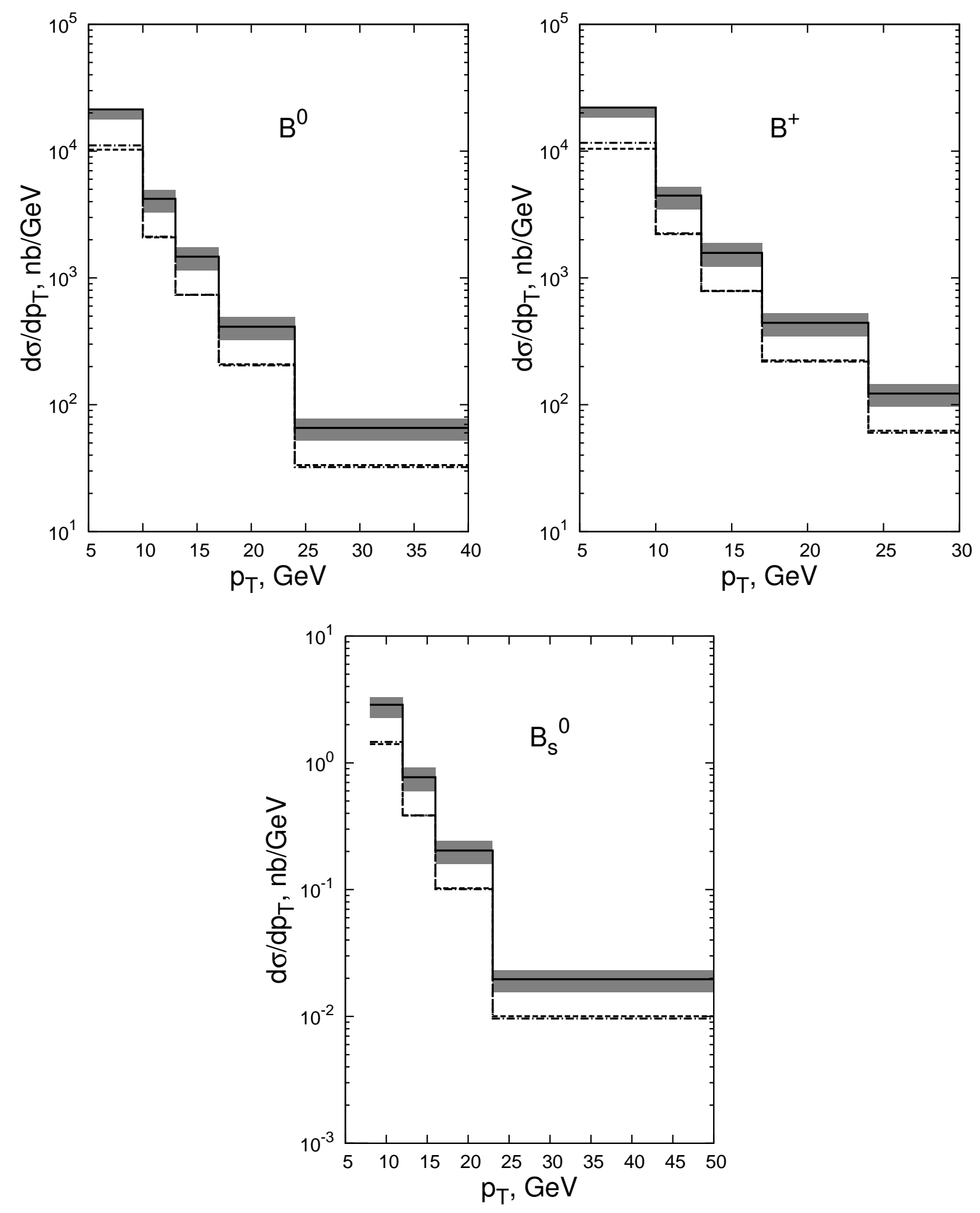

FIG. 5: Theoretical predictions for the transverse momentum distributions of $B^{0}$ (top), $B^{+}$(middle), $B^{s}$ (bottom) mesons in $p p$ scattering at $\sqrt{S}=14 \mathrm{TeV}$ and $|y|<1.0$ obtained in the LO PRA. The notations as in the Fig. 4. 

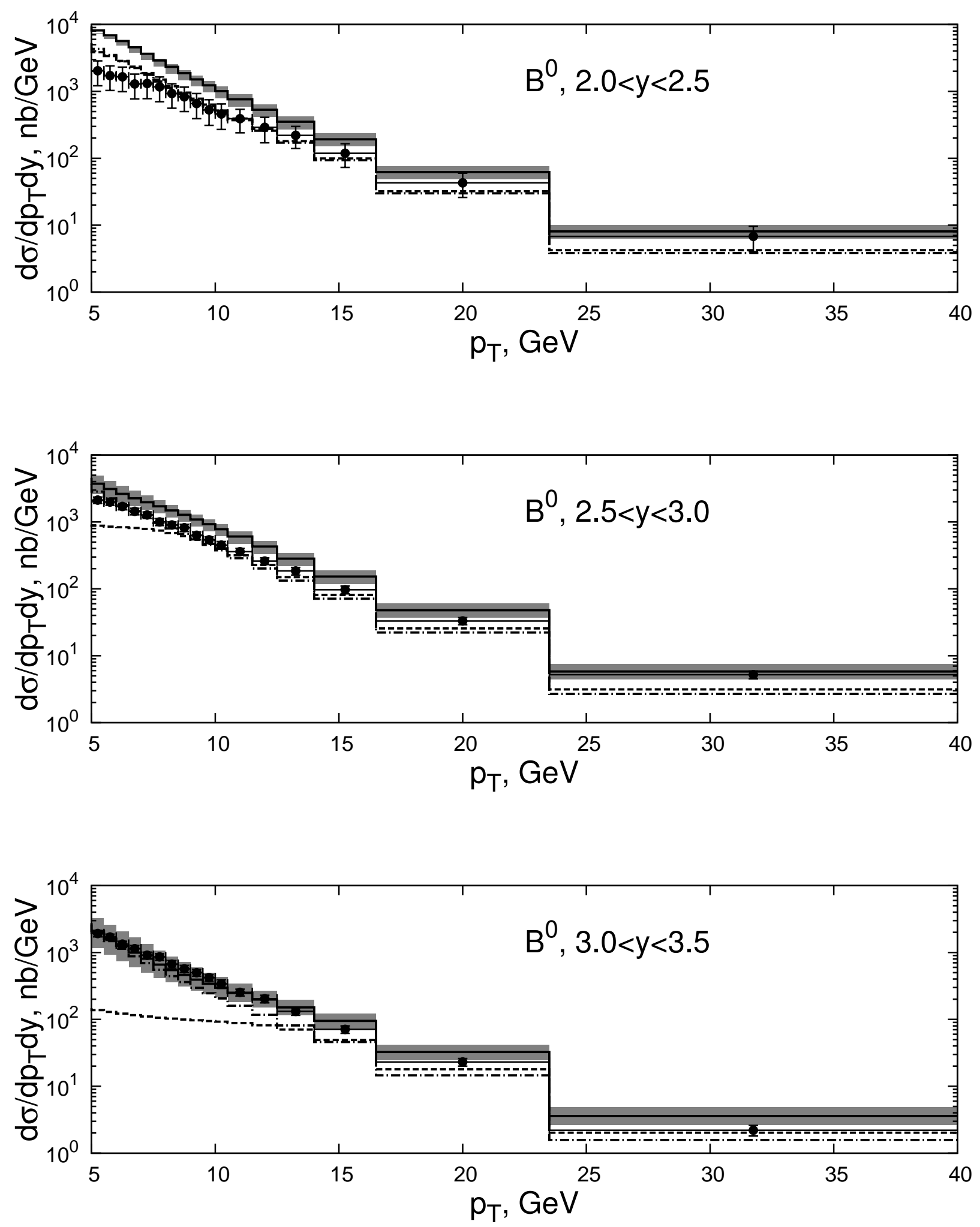

FIG. 6: Transverse momentum distributions of $B^{0}$ mesons in the forward rapidity regions of $2.0<y<2.5$ (top), $2.5<y<3.0$ (middle), and $3.0<y<3.5$ (bottom) in $p p$ scattering with $\sqrt{S}=7 \mathrm{TeV}$. The LHCb data at LHC are from the Ref. [8]. The notations as in the Fig. 4] 

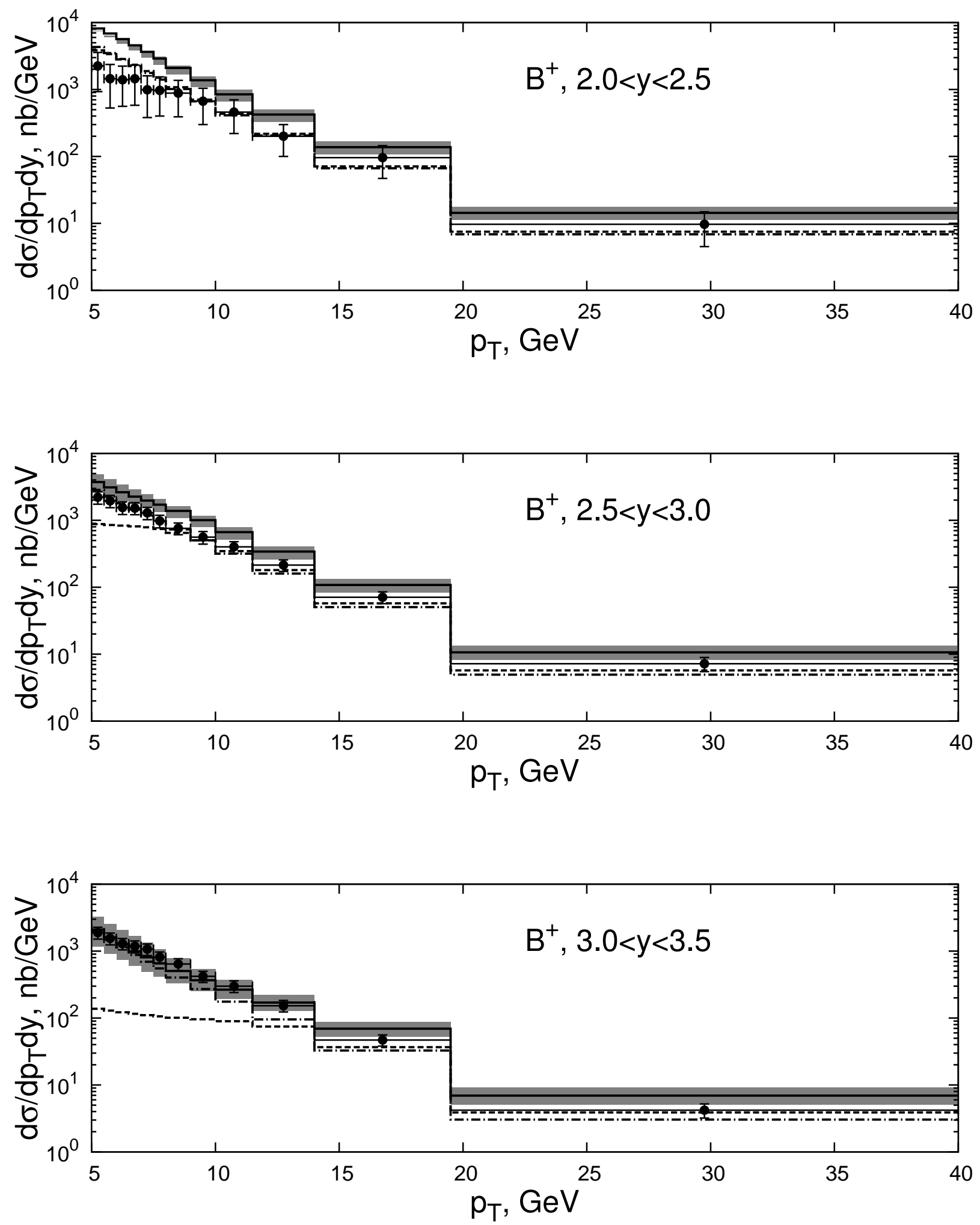

FIG. 7: The same as in the Fig. 6 for $B^{+}$mesons 

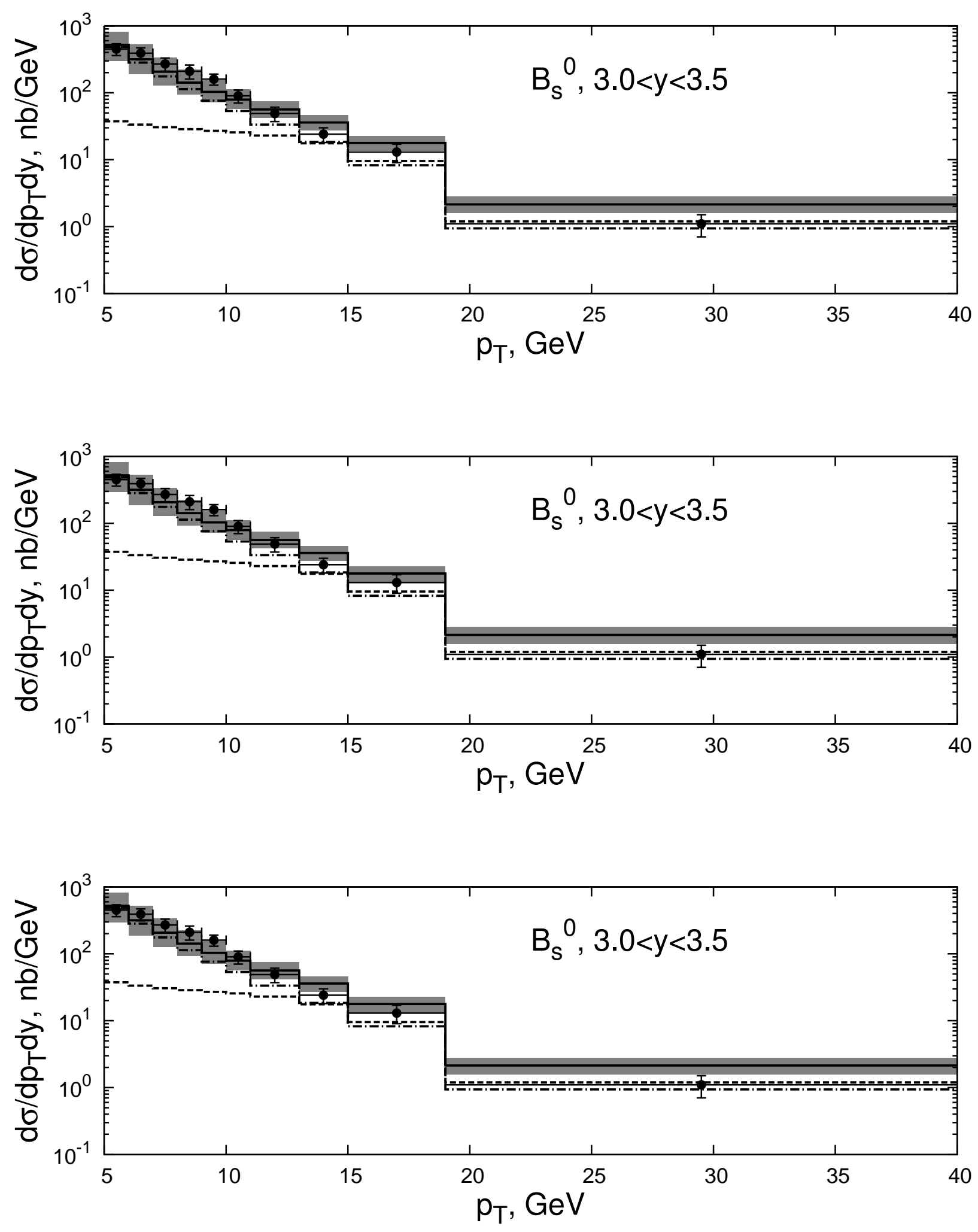

FIG. 8: The same as in the Fig. 6 for $B_{s}^{0}$ mesons 

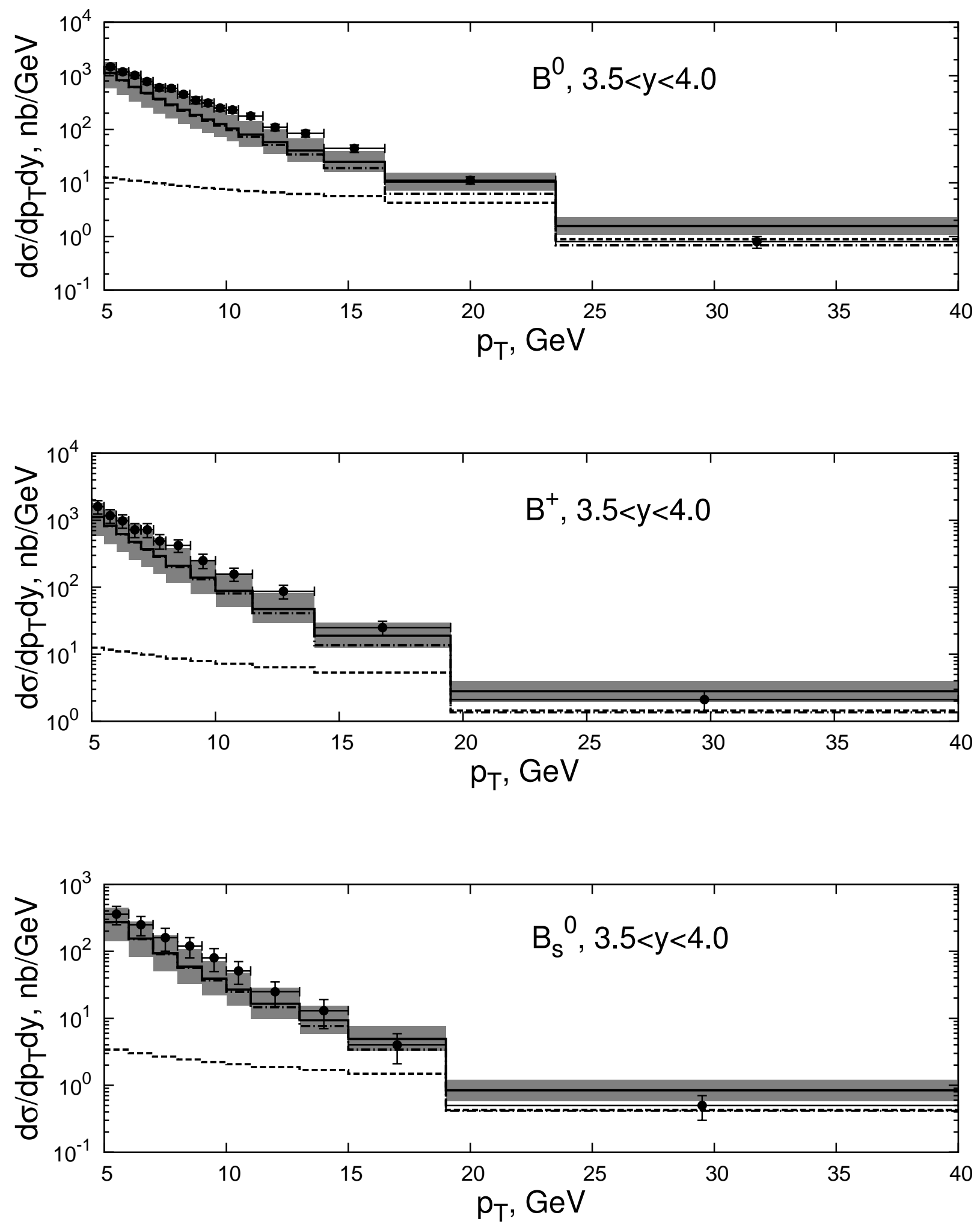

FIG. 9: Transverse momentum distributions of $B^{0}, B^{+}$, and $B_{s}^{0}$ mesons in the forward rapidity region of $3.5<y<4.0$ in $p p$ scattering with $\sqrt{S}=7 \mathrm{TeV}$. The LHCb data at LHC are from the Ref. [8]. The notations as in the Fig. [4, 

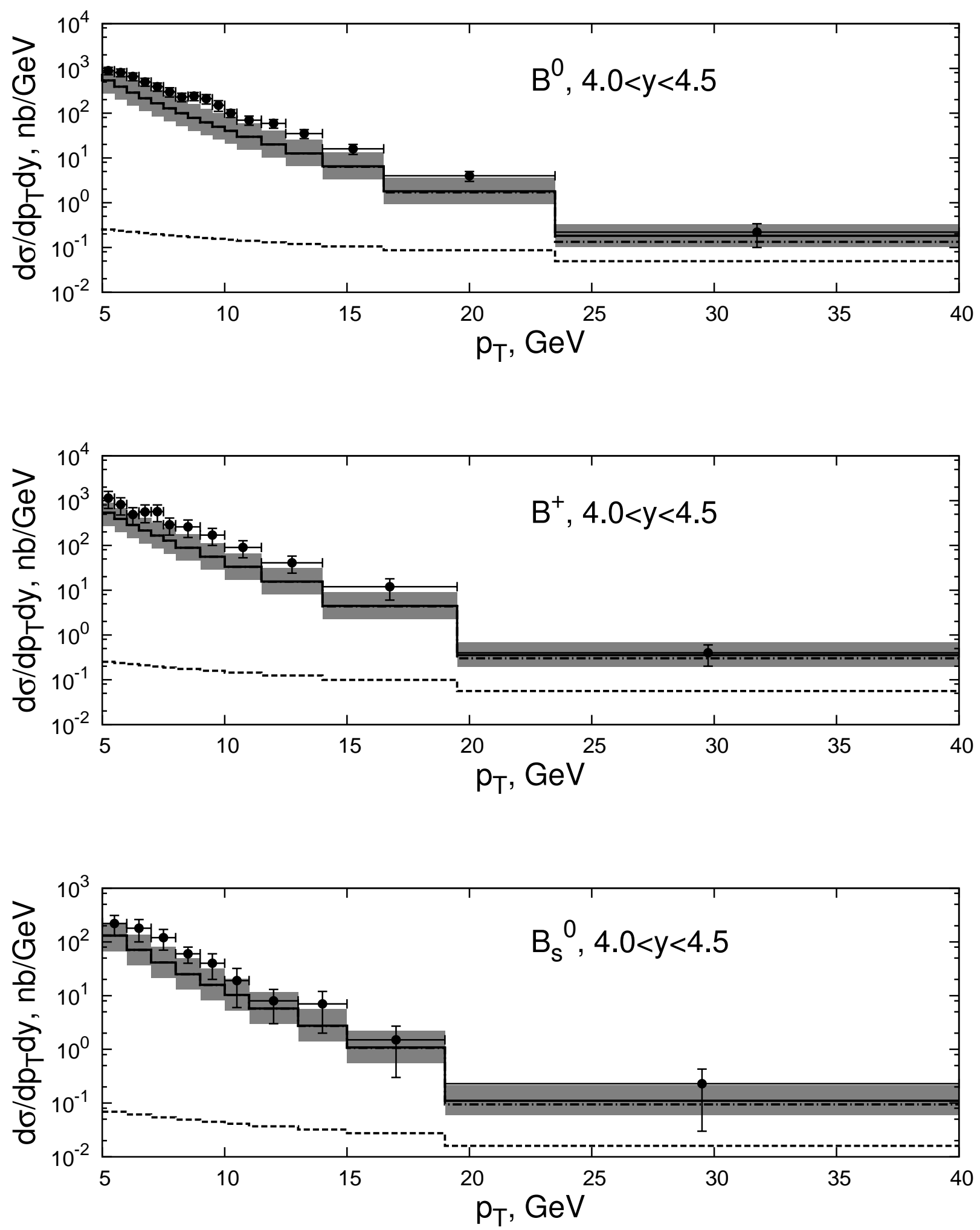

FIG. 10: The same as in the Fig. 9 for $4.0<y<4.5$. 

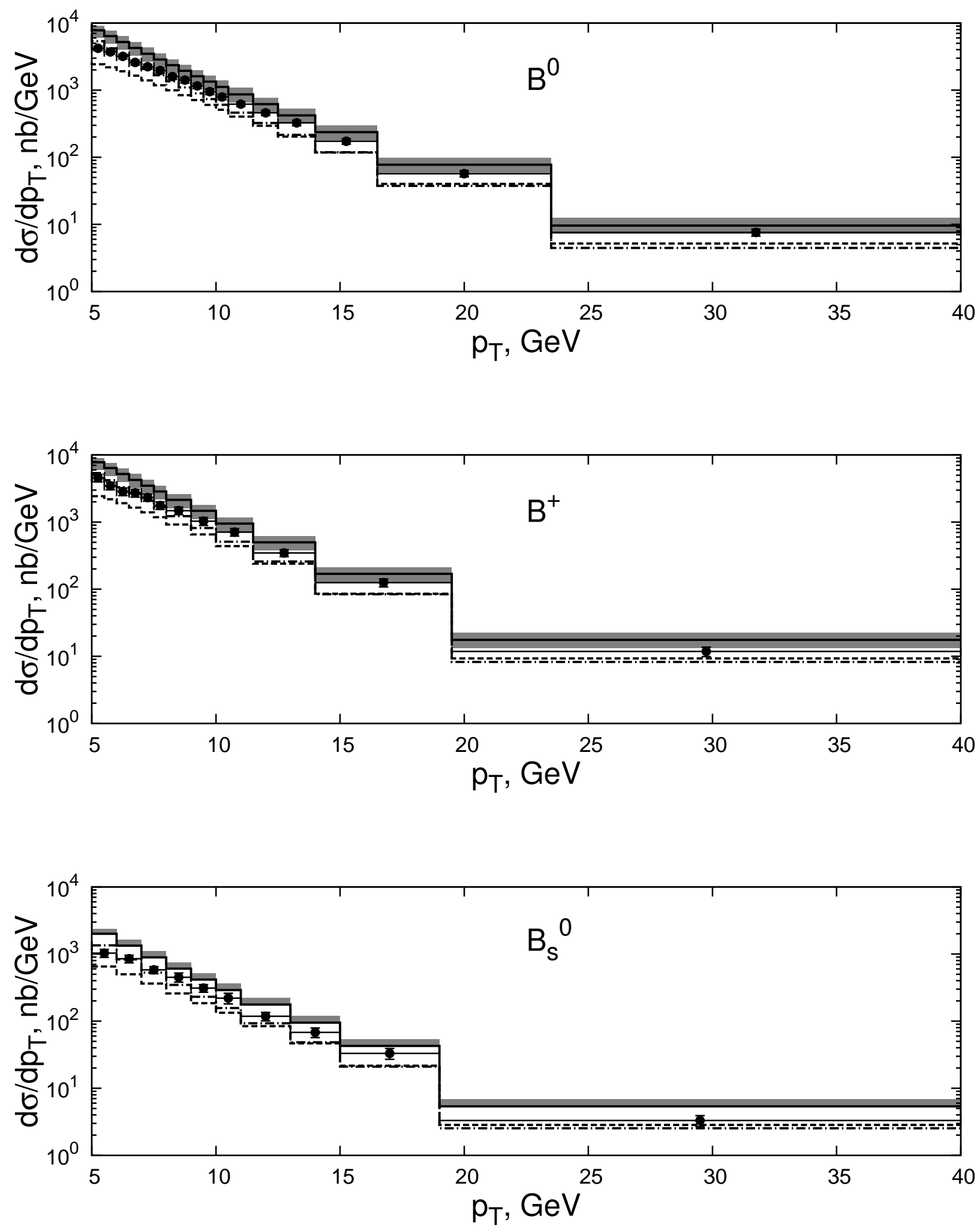

FIG. 11: Transverse momentum distributions of $B^{0}$ (left-top), $B^{+}$(right-top), $B_{s}^{0}$ (bottom) mesons in the forward rapidity region in $p p$ scattering with $\sqrt{S}=7 \mathrm{TeV}$ and $2.0<y<4.5$. The LHCb data at LHC are from the Ref. [8]. The notations as in the Fig. 4. 